\title{
Research on Applications of Cell-Free Protein Synthetic System in the Field of Biopharmaceutical Engineering
}

\author{
Wei Long, Weihua Tang, Chunhui Lv
}

Tinjin Modern Vocational Technology College, Tianjin, China, 300350)

\begin{abstract}
Keywords: cell-free protein synthesis system, biopharmaceutical engineering, industrial production
\end{abstract}

\begin{abstract}
As one of the techniques of protein synthesis in vitro, the cell-free protein synthetic system has many advantages, such as simple manipulation, good genetic background and so on. In recent years, the technology of cell-free protein synthetic system has made some breakthroughs in the fields of stability of amino acid and protein folding in vitro. Based on these technologies, this paper discusses the applications of cell-free synthetic expression system in the field of biopharmaceutical engineering to provide some references for the relative researchers.
\end{abstract}

\section{Concept of cell-free protein synthetic system}

With the development of biotechnology, protein expression technology has been mastered by most researchers. The routine application of protein expression technique is usually used in Escherichia coli, yeast, mammalian cells and insect cell. It was transformed or transfected by plasmid, the nucleic acid into the cell, then the use of amino acid residues, ribosomes and intracellular enzyme to protein synthesis. In these expression systems, because of the advantages of convenient culture, short protein synthesis time, low cost and high efficiency, it is widely used. But the limitations and shortcomings of Escherichia coli expression system is also very significant, the problem has also been plagued and hinder researchers to obtain ideal protein to carry out research, urgently needs a better way to synthesis protein. Cell-free protein synthesis system as a rapid and efficient in vitro protein synthesis by means of synthetic enzymes in the cell extract of the complement of transcription and translation of the required material substrate and energy to complete target protein. Compared with the traditional living cells in vivo expression system based on cell-free protein synthesis system has many advantages, such as no membrane barrier, gene polymerase concentration control, quantitative measurement and high throughput.

\section{Technical progress of cell-free protein synthetic system}

It needs to consume a lot of energy in the process of protein synthesis. The research shows that the reaction time for energy shortage will directly reduce the cell-free protein synthesis system and reduce the production efficiency of the system, so the choice of appropriate energy supply system is essential for improving the production efficiency of the system. A series of studies found that the use of cell-free protein synthesis system protein synthesis, amino acid will be consumed due to reaction termination in the reaction starting within $30 \mathrm{~min}$, supplemented by the corresponding 
amino acid can prolong the duration of the reaction system, improve the yield of protein. They further simplify the preparation process by low speed centrifugation and shorten the reaction time of runoff, which greatly saves time and reduces costs. Recent studies have shown that by changing the type of ions in the system, the use of bead grinding method or ultrasonic fragmentation method to replace the traditional high-pressure homogenization method can improve the production efficiency of cell-free protein synthesis system, and further reduce production costs. These results suggest that the presence of an unknown enzyme in cell-free protein synthesis system may degrade the amino acids of the exogenous addition. In order to stabilize the substrate supply, the cell-free protein synthesis system was used to reconstruct the genome of the plant, which was used to prevent the rapid degradation of amino acids without affecting the growth of the bacteria. It will consume a large amount in the initial stage of the reaction; secondly deduced amino acids may lead to degradation of the enzyme, such as arginine, tryptophan decarboxylase enzyme and serine deaminase. Finally, the corresponding genes walk into the genome of knockout. The improved cellfree protein synthesis system promoted the stability of amino acid, prolonged the reaction time and increased the production efficiency.

The correct folding of proteins is to protect the different hydrophobic regions of the target protein and the two do not interfere with each other, and provide a suitable chemical environment. In addition, the addition of cofactors, such as iron sulfur clusters, and so on, to promote the isomerization and formation of two disulfide bonds. After a long period of natural evolution, biological cells are not in the same region to complete oxidative folding and protein synthesis. The cell-free system improves its drawbacks and can be done in the same area. The gel has a controlled and binding effect, reducing the release of multiple strands to prevent aggregation precipitation. There are many factors involved in protein folding, however, in the cell-free system, it is necessary to construct an environment similar to that expressed in the cell to ensure the correct folding of the protein. In vivo, there are molecular chaperones, such as proteins, to help maintain the correct conformation of the nascent polypeptide chain and avoid the aggregation of proteins. The complex protein was successfully expressed by the direct addition of molecular chaperones in cell-free system. In addition to simply directly into the molecular chaperone, by adding amphoteric polysaccharide gel in a cell-free system to help protein folding, the gel can be combined and control the polypeptide chain release so as to avoid excessive protein expression leads to aggregation. There are many factors that affect the folding of proteins. The essential means to help correct the folding of proteins in cells is to try to simulate the aggregation of proteins in the cell. There are many factors that influence the folding of proteins. The essential way to help protein folding is to try to simulate the real environment of protein expression in cells.

\section{Applications of cell-free protein synthetic system in biopharmaceutical engineering}

In the post-genomic era, a major challenge for scientists is to systematically elucidate gene encoding the whole protein structure, function and interactions. Therefore, the expression of technology is very important to establish a high throughput protein efficiently. In recent years, cellfree protein synthesis system has been popular for its unique advantages. The in vivo expression 
system compared with traditional cell-free protein synthesis system, you can directly use the fragment as template, eliminates the time-consuming process of molecular cloning; secondly, some difficult in complex protein in vivo expression system, such as membrane proteins and protein structure of the cysteine rich complex, correctly folded in cell-free protein synthesis system; at the same time, through the transformation of the protein in the process of translation, can obtain the special marker protein. The traditional preparation technology need to purify large quantities of soluble protein, and then modified glass slides with covalent or immobilization in order to achieve. The use of cell-free protein synthesis system can be achieved in one step of protein expression and immobilization, eliminating the time-consuming and costly molecular cloning, protein expression and purification process. Moreover, cell-free protein synthesis system is an open and flexible method, which can be used to realize the specific elevation flux protein expression in the process of protein translation. The cell-free protein expression system has many advantages, which is conducive to the establishment of high-throughput system. First of all, fragments in the system can directly as a template to simplify the cloning process; secondly, efficient system trace protein expression has been developed; thirdly, the automatic operation can be realized; finally, membrane structure does not exist in the reaction system, the reaction process, the implementation of external intervention immediately. High throughput technology provides a good research platform for researchers, which can not only make proteomics chips, but also realize the direct expression of drug target proteins.

The so-called complex refers to the virus like particles. Because its structure is similar to that of virus particles, it can trigger immune response, but the virus like particles do not carry genetic material, so it cannot achieve self-replication. In general, the viral capsid protein with natural selfassembly ability, can form a hollow structure in prokaryotic system, eukaryotic system, is highly concerned by researchers. Bundy et al. successfully expressed coat protein in cell-free system. The characteristics of the cells themselves, increasing the scope of the use of virus like particles. Swartz and Bundy restored the environment control. The expression of capsid protein monomer two disulphide bonds was adjusted to promote the stability of virus like particles. Virus-like particles (VLPs) is a complex assembled by one or more proteins, which is similar to the structure of virus particles. It does not contain infectious genetic material. VLPs has a true virus capsid protein conformation, causing cell mediated immune response in the body, and overcomes the shortcomings of existing vaccines raised the possibility, is a safe and effective vaccine subunit. Until now, scientists have successfully developed thirty kinds of VLPs for the treatment of viral diseases, including hepatitis virus vaccine and human papilloma virus vaccine. It has been approved and widely used in the world. It is difficult to find a balance between the two points. As a result, many VLPs can only be used in small-scale basic research due to their remarkable clinical effect.

A small number of mammalian genomes are made up of membrane proteins, and more than half of the genome can be used as target cells. Therefore, it is of great significance to study the structure and function of membrane proteins. At present, there are some problems in the expression of membrane proteins. Overexpression of membrane protein in vivo can easily lead to precipitation accumulation and produce biological toxicity; structure of bacterial cell phospholipid membrane 
will affect mammalian membrane protein folding and insertion; membrane proteins from mammalian cells after isolation and purification treatment make it into artificial lipid is very difficult. These factors greatly increase the difficulty of purification and recombinant expression of membrane proteins. The cell-free system can provide an efficient platform for the purification and expression of membrane proteins. The liposome and the detergent are directly typed into the open cell cell-free protein expression system, and the protein can be directly entered into the hydrophobic environment after the expression, or can be integrated into the liposome to prevent aggregation. Lactic acid membrane protein differs from membrane proteins of bacteria. The psychrophilic bacteria can maintain the integrity and function of the structure of the catalyst under low temperature conditions. When the temperature of the cells was reduced, the lactic acid bacteria cells could synthesize a group of cold adapted proteins, which could maintain the normal biological function of the cells and then enhance the tolerance to cold and increase the survival rate. Twodimensional gel electrophoresis can be used to separate cell membrane proteins. The bacteria adapt to cold, or make the stress response, the adaptive regulation, leading to changes in cell membrane fatty acid and membrane protein expression, thereby reducing the damage caused by cold bacteria. However, the changes of cell membrane fatty acids were mainly due to the increase of unsaturation, the decrease of the length of carbon chain, the increase of methyl branches, and the occurrence of branching structures. Therefore, it does not belong to cold shock proteins, and the characteristics and functions of its specific expression need to be further studied.

The cell lymphoma is a solid tumour that is caused by the malignant proliferation of cells. The immune system cannot recognize abnormal cells. It is worth noting that the variable region of immunoglobulin cell surface expression of malignant, namely idiotype is specific. This provides a good target for the treatment of cell lymphoma, which can be used to suppress the tumour by activating the immune system by injecting the vaccine prepared by the idiotype protein. In order to enhance the immunogenicity of idiotype proteins, the unique proteins of tumour cells are fused with granulocyte macrophage colony stimulating factor, and no other carrier proteins and adjuvants are needed. Clinical data show that the vaccine can effectively activate the immune system to control tumour cells. However, each cell lymphoma patient carries a unique type of immunoglobulin is not the same, so the need for different patients individually tailored vaccine, which is a major challenge for vaccine production. The traditional method of using human cells as bioreactor to produce vaccine is not only long cycle, but also expensive, which is not conducive to the popularization and application of cell lymphoma vaccine. If the tumour vaccines use individual customization of production, so the whole process will be directly from the patient's disease diagnosis to vaccine production, eliminating the need for intermediate cycle long cell culture stage. It reduces the risk of cross contamination between patients with drugs. In the early days of the mice, the vaccine was effective in inhibiting the growth of tumour cells. These results indicate that cell-free protein synthetic system has a great potential in the field of tumour individualized therapy. 


\section{Conclusion}

The technical progress of cell-free protein synthetic system provides a good platform for the development of biological research and biopharmaceutical engineering. The high-throughput expression, complex expression, membrane protein expression and immunoglobulins expression gradually show the application value in the field of biological pharmacy and the production of small molecule natural products. We believe that the cell-free protein synthetic system will play an increasingly important role in the field of biological pharmacy.

\section{References}

[1] Yang Yingchao, Xin Xiaofang, Progress in Cell-free Protein Synthesizing System, Chinese Pharmaceutical Affairs, 25(8), pp. 832-835, 2011.

[2] Jia Xiaoge, Deng Zixin, Liu Tiangang, Progress of cell-free protein synthesis system and its applications in pharmaceutical engineering-A review, 56(3), pp. 530-542, 2016.

[3] Sheng Jiayuan, Zhang Xu, Zheng Qiang, Xu Zhinan, Cell-free protein synthetic system: progress and applications in biopharmaceutical engineering, Chinese Journal of Biotechnology, 30(10), pp. 1491-1503, 2014.

[4] Lu Linli, Liu Bicheng, The Cell-free Protein Synthesis-based Protein Microarray Technology, Journal of Biomedical Engineering, 27(6), pp. 1397-1400+1409, 2010. 